\title{
Searches for dark matter production with ATLAS (MET+X)
}

\author{
Ruth Pottgen*† \\ Stockholm University (SE) \\ E-mail: ruth.poettgen@cern.ch
}

Dark Matter constitutes more than $80 \%$ of the matter in the universe but cannot be accommodated within the Standard Model of particle physics. The search for Dark Matter is one of the main objectives of the LHC physics programme. This contribution summarises searches performed in final states with large missing transverse momentum using $3.2 \mathrm{fb}^{-1}$ of data at a centre-of-mass energy of $13 \mathrm{TeV}$ collected by the ATLAS detector in 2015.

Fourth Annual Large Hadron Collider Physics

13-18 June 2016

Lund, Sweden

* Speaker.

On behalf of the ATLAS Collaboration 


\section{Introduction}

Various cosmological observations on largely different scales - ranging from single galaxies up to the entire universe - provide compelling evidence for the existence of a non-luminous form of matter which has at most very weak interactions with normal matter and might even interact only gravitationally. This dark matter (DM) is estimated to amount to about $26 \%$ of the total matterenergy-content of the universe - about five times as much as the amount of normal matter, i.e. stars, gas, dust etc. [1]. The Standard Model (SM) of particle physics does not provide a viable candidate to account for all of this additional matter and hence its existence is a clear evidence for physics beyond the Standard Model (BSM). A popular class of candidates of DM particles are weakly interacting massive particles (WIMPs) which are assumed to be a thermal relic from the early universe and would have masses and interaction strengths that naturally yield the observed abundance of DM. There is a broad search programme for such particles and the experiments can be grouped into three categories: Direct searches aim at detecting the recoil of nuclei in a target material due to WIMPs scattering off the nuclei, indirect searches look for annihilation of WIMPs into SM particles and collider experiments mostly search for hints of DM being produced in the collisions of SM particles. If WIMPs are produced in the collision, they escape undetected and are only visible indirectly: If there is another object in the event that can be used to trigger on, the presence of invisible particles manifests itself as an imbalance in the total transverse momentum, denoted $E_{\mathrm{T}}^{\text {miss }}$. There is a wide range of such $E_{\mathrm{T}}^{\text {miss }}+X$ (or mono- $X$ ) searches at the collider experiments and this contribution will focus on the ATLAS [2] results in these channels obtained with the 2015 data set of $3.2 \mathrm{fb}^{-1}$ at a centre-of-mass energy of $13 \mathrm{TeV}$.

\section{Models and Presentation of Results}

During the LHC run-1, an effective field theory (EFT) was used to derive limits on WIMP production in a model-independent way [3, 4]. In such an effective theory it is assumed that there is a heavy particle which mediates the interaction between SM particles and WIMPs, but this mediator is too heavy to be produced directly in the collisions. It can therefore be integrated out and the theory is left with only two parameters: the mass of the WIMP $\left(m_{\chi}\right)$ and the suppression scale $\left(M_{*}\right.$ or $\Lambda$ ). While this approach allowed to illustrate the complementarity of collider results and those from direct and indirect searches, a detailed discussion arose around the question of the validity of the EFT as a low-energy approximation at LHC energies. By the end of run-1, several searches therefore included a truncation procedure in the presentation of their results to illustrate the validity of the EFT approach. In this truncation, events with a momentum transfer larger than $g M_{*}{ }^{1}$, are removed from the signal simulation and the limits are recomputed. This is done for several values of the coupling $g$. As a further step towards a UV-complete theory, the use of simplified models was introduced already at the end of run-1 and the ATLAS\&CMS DM forum (now LHC DM working group) benchmarked a number of such models for use in run-2 [5, 6]. These models assume that WIMPs are Dirac-fermions. There are mostly four parameters: the masses of the mediator $\left(M_{\mathrm{Med}}\right)$ and the WIMP, and the two couplings of the mediator to DM ( $g_{\mathrm{DM}}$ or $\left.g_{\chi}\right)$ and SM particles $\left(g_{\mathrm{q}}\right.$ or $g_{\mathrm{SM}}$ ), respectively. For most of the searches outlined in Sec. 3 the couplings are set to $g_{\chi}=1$

\footnotetext{
${ }^{1}$ The symbol $g$ represents the product of the couplings of the mediator to WIMPs and SM particles, respectively
} 
and $g_{\mathrm{q}}=0.25$. Different types of mediators (e.g. vector or axial-vector) with minimal widths are considered. While the number of parameters in these models is higher than in the EFT approach, there are no concerns about their validity.

\section{Searches}

ATLAS performed a number of $E_{\mathrm{T}}^{\text {miss }}+\mathrm{X}$ searches with the 2015 dataset, with the object $\mathrm{X}$ being a jet, a photon, a heavy vector boson or a Higgs boson. The main challenge for these analyses is the precise estimation of the dominant backgrounds. To achieve this, data control regions (CRs) are used and in most cases a simultaneous fit to all regions is performed to normalise the backgrounds. Sub-dominant backgrounds are in most cases estimated from simulation, with typical exceptions being multi-jet and non-collision backgrounds that are instead estimated in data-driven ways. Most of the searches use the missing transverse energy as the discriminating variable and look for an excess over the SM prediction in regions of large $E_{\mathrm{T}}^{\text {miss }}$. In the absence of an excess limits are set on the model parameters mentioned in Sec. 2.

\subsection{Mono-Jet}

The traditional workhorse among the mono-X searches is the mono-jet search [7], where the WIMPs are assumed to be produced together with a jet from initial state radiation (ISR). The events are selected with a $E_{\mathrm{T}}^{\text {miss }}$ trigger with a threshold of $70 \mathrm{GeV}$. Up to four jets with a transverse momentum above $30 \mathrm{GeV}$ are allowed in the event and the leading jet must have $p_{\mathrm{T}}>250 \mathrm{GeV}$. To suppress contributions from multi-jet events, all jets have to be well separated from the direction of the $E_{\mathrm{T}}^{\text {miss }}$, i.e. the azimuthal distance $\Delta \phi$ between the any jet and $E_{\mathrm{T}}^{\text {miss }}$ must be greater than 0.4. The $E_{\mathrm{T}}^{\text {miss }}$ is required to be greater than $250 \mathrm{GeV}$ at least and several signal regions (SRs) are defined by different further cuts on $E_{\mathrm{T}}^{\text {miss }}$ at $300 \mathrm{GeV}, 350 \mathrm{GeV}, 400 \mathrm{GeV}, 500 \mathrm{GeV}, 600 \mathrm{GeV}$ and $700 \mathrm{GeV}$. Inclusive regions, i.e. all events with $E_{\mathrm{T}}^{\text {miss }}$ greater than a given value, are used to provide model independent limits while exclusive regions (bins in $E_{\mathrm{T}}^{\text {miss }}$ defined by the cuts above) are used to extract limits on the DM model considered: An axial-vector mediator $(A)$ with the standard coupling assumptions (see Sec. 2).

The production of $Z$-bosons with additional ISR jets where the $Z$ decays into a neutrino-antineutrino pair is an irreducible background to this search as it results in exactly the same signature. Further large contributions arise from $W+$ jets production with leptonic $W$ decays if the decay lepton is not reconstructed or if it is a hadronically decaying $\tau$. These backgrounds are estimated using three orthogonal data control regions: A $W(\mu v)+$ jets CR is defined by selecting events with exactly one muon, no electrons and a transverse mass $m_{\mathrm{T}}^{2}$ between $30 \mathrm{GeV}$ and $100 \mathrm{GeV}$; For the $W(e v)+$ jets $\mathrm{CR}$ exactly one electron and zero muons are required (no cut on the transverse mass is applied); The $Z(\mu \mu)+$ jets $\mathrm{CR}$ requires exactly two muons with an invariant mass $m_{\ell \ell}$ between $66 \mathrm{GeV}$ and $116 \mathrm{GeV}$ and zero electrons. In addition to these selections, the same cuts as for the SRs are also applied in the CRs. The $E_{\mathrm{T}}^{\text {miss }}$ distributions in the CRs with the cut at $250 \mathrm{GeV}$ are shown in Fig. 1, the $W(\mu v)+$ jets CR on the top left, the $W(e v)+$ jets CR on the top right, the $Z(\mu \mu)+$ jets $\mathrm{CR}$ on the

\footnotetext{
${ }^{2}$ The transverse mass is calculated as $m_{\mathrm{T}}=\sqrt{2 p_{\mathrm{T}}^{\ell} E_{\mathrm{T}}^{\text {miss }}\left(1-\cos \Delta \phi\left(\ell, E_{\mathrm{T}}^{\text {miss }}\right)\right)}$ using the transverse momentum of the lepton $\left(p_{\mathrm{T}}^{\ell}\right)$, the missing transverse energy and the angle between the two.
} 

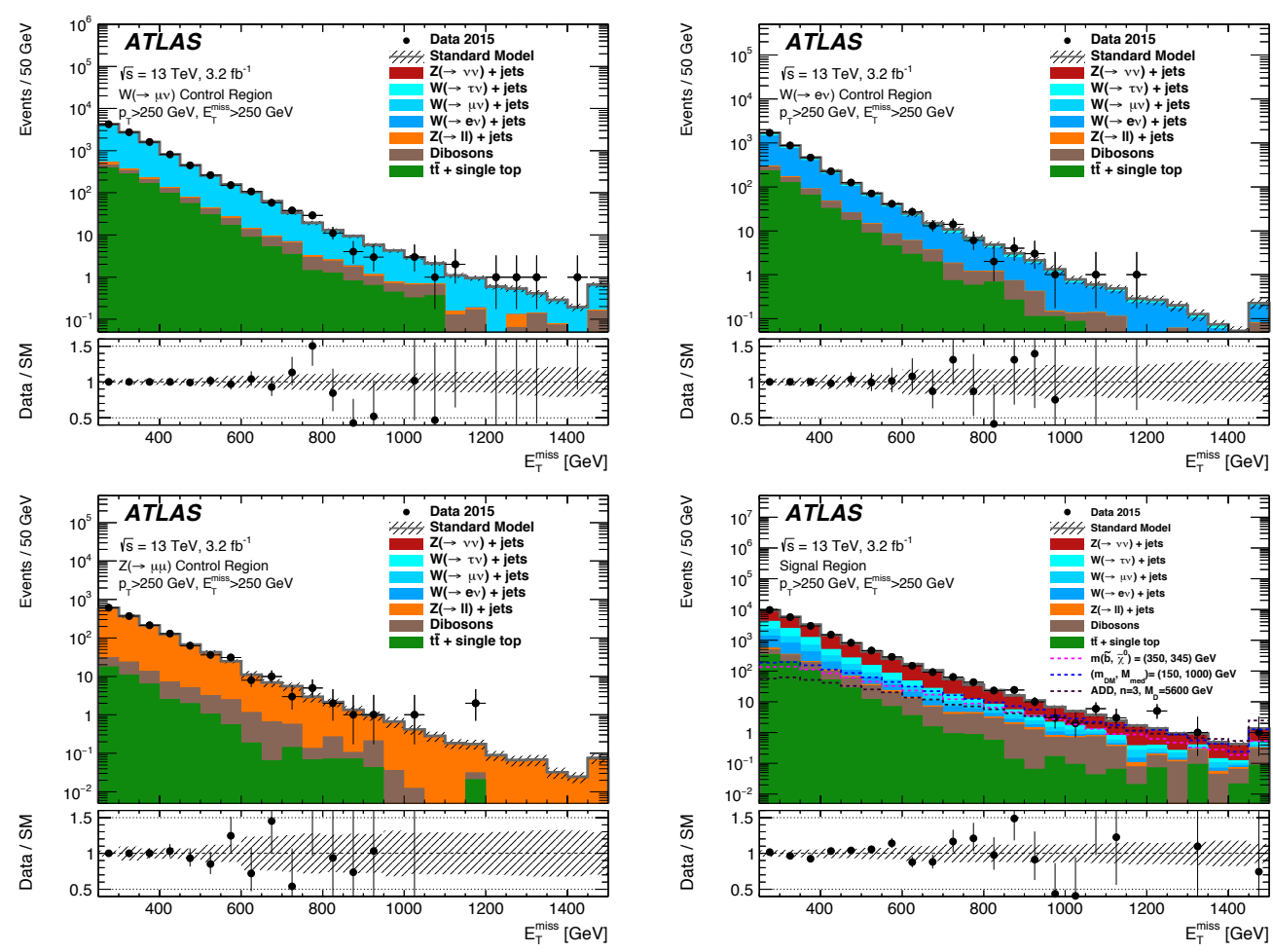

Figure 1: $E_{\mathrm{T}}^{\text {miss }}$ distribution in the lowest inclusive control and signal regions. The $W(\mu v)+$ jets $\mathrm{CR}$ on the top left, the $W(e v)+$ jets $\mathrm{CR}$ on the top right, the $Z(\mu \mu)+$ jets $\mathrm{CR}$ on the bottom left and the signal region on the bottom right. Below each distribution the ratio of data to prediction is shown, the error bands include the statistical and experimental uncertainties in the background prediction [7].

bottom left. Good agreement is observed between data and SM prediction which gives confidence in the background modelling.

The $E_{\mathrm{T}}^{\text {miss }}$ distribution in the most inclusive signal region after the fit is shown in the bottom right panel of Fig. 1. The dominant uncertainties are coming from uncertainties in the transfer from $W+$ jets CRs to the $Z(v v)+$ jets estimate $(2-4 \%)$, theory uncertainties in the top background (3\%) and statistical uncertainties due to the limited event numbers in the CRs $(<10 \%$ even in the highest regions). No significant excess is observed and $95 \%$ confidence level (CL) limits are derived in the plane of WIMP mass and mediator mass, as shown in the left panel of Fig. 2. The dark-red solid line indicates the observed limit, the dashed red lines the $\pm 1 \sigma$ signal theory uncertainties. The dashed blue line with the yellow band is the expected limit with the $\pm 1 \sigma$ uncertainty. The light-red line indicates the mass values for which the observed relic density is obtained in this model. The region excluded due to perturbativity, defined by $\left.m_{\chi}>\sqrt{(} \pi / 2\right) m_{A}$, is indicated by the grey area. In the region where the mediator can be produced on-shell, mediator masses up to approximately $1 \mathrm{TeV}$ are excluded for WIMP masses up to roughly $250 \mathrm{GeV}$. In the off-shell regime the decay into WIMPs is kinematically suppressed and the analysis loses sensitivity.

In the right panel of Fig. 2 the limits have been translated into 90\%CL limits on the spin-dependent WIMP-proton scattering cross section and are compared to several direct-detection experiments. While this comparison strictly is only valid within the model used in this analysis, it illustrates 

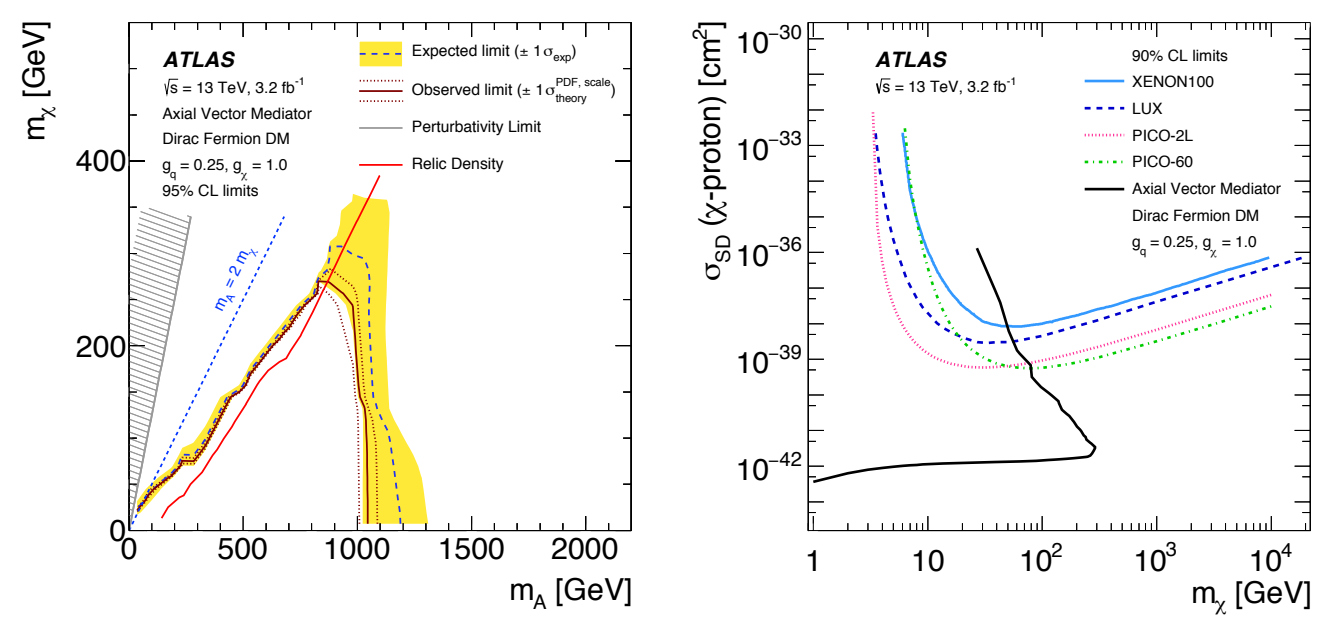

Figure 2: Left: 95\% CL exclusion contours in the $m_{\chi}-m_{A}$-plane. The observed limit (with the $\pm 1 \sigma$ theory uncertainties on the signal cross section) is shown by the dark-red solid (dashed) line. The expected bounds with the $\pm 1 \sigma$ uncertainties are indicated by the blue dashed line with the yellow band. The light-red line indicated mass values for which the observed relic density is obtained. The grey area illustrates the region excluded by perturbativity $\left.\left(m_{\chi}>\sqrt{(} \pi / 2\right) m_{A}\right)$. Right: $90 \%$ CL limits on the spin-dependent WIMP-proton scattering cross section compared to results from direct-detection experiments [7].

the complementarity of the different approaches: The collider experiments can set considerably stronger bounds at low WIMP masses where the direct-detection searches are not sensitive.

\subsection{Mono-Photon}

The mono-photon search [8] selects events by a single-photon trigger with a $p_{\mathrm{T}}$ threshold of $120 \mathrm{GeV}$. The $p_{\mathrm{T}}$ of the offline reconstructed photon must be greater than $150 \mathrm{GeV}$, the same cut is applied for $E_{\mathrm{T}}^{\text {miss }}$. At most one jet with a $p_{\mathrm{T}}$ greater than $30 \mathrm{GeV}$ is allowed in the event and the $E_{\mathrm{T}}^{\text {miss }}$ must be separated from the jet and the photon by $\Delta \phi\left(E_{\mathrm{T}}^{\text {miss }}, \gamma\right.$ or jet $)>0.4$.

Analogously to the mono-jet search, the main backgrounds are $Z(v v)+\gamma$ and $W(\ell v)+\gamma$. They are constrained by means of data control regions defined by selecting one or two leptons explicitly and applying mass cuts consistent with the boson mass. A dedicated control region is used to constrain the $\gamma+$ jet background: The $E_{\mathrm{T}}^{\text {miss }}$ is required to be between $85 \mathrm{GeV}$ and $110 \mathrm{GeV}$ and the azimuthal separation of $E_{\mathrm{T}}^{\text {miss }}$ and the photon has to satisfy the condition $\Delta \phi\left(E_{\mathrm{T}}^{\text {miss }}, \gamma\right)<3$.

In the signal region, electrons and muons are vetoed. The $E_{\mathrm{T}}^{\text {miss }}$ distribution in this region is shown in the left panel of Fig. 3. Within uncertainties the simulation agrees with the data, with the dominant uncertainty coming from the limited statistics in the data control regions and amounting to $9 \%$ (the total uncertainty is 11\%). Limits are derived on the same DM model as in the mono-jet search. The 95\% CL exclusion contours in the $m_{\chi}-m_{A}$-plane are shown in the right panel of Fig. 3: The observed limit is displayed as a black solid line with the $\pm 1 \sigma$ theory uncertainty indicated by the red band. The expected limit with the $\pm 1 \sigma$ uncertainty is given as the dotted black line with a green band. In the on-shell regime mediator masses up to $700 \mathrm{GeV}$ are excluded. Mass combinations corresponding to the observed relic density are indicated by the dotted pink line.

Apart from the WIMP pair production with a photon from ISR, this analysis is also sensitive to 

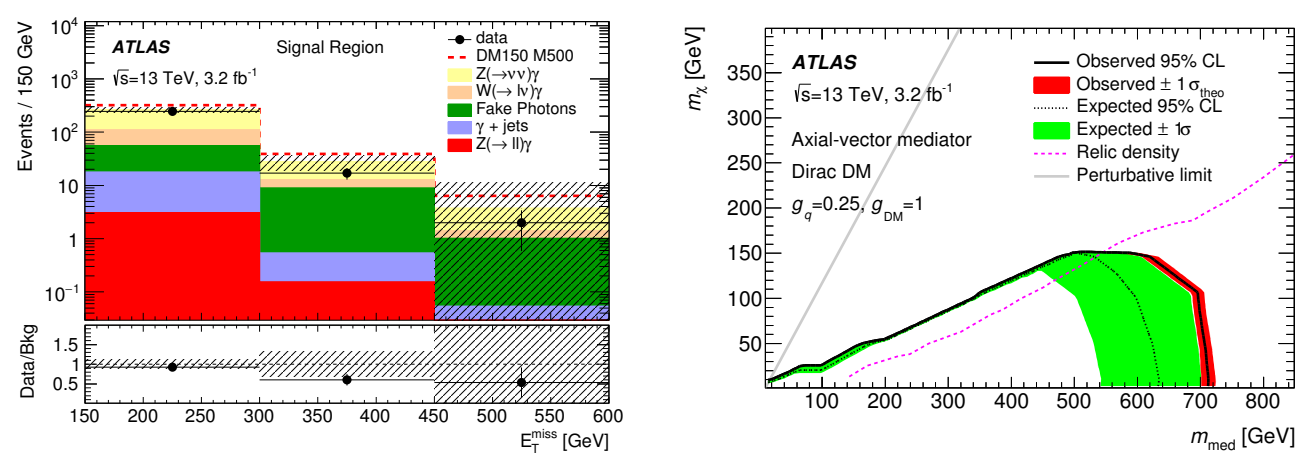

Figure 3: Left: Distribution of $E_{\mathrm{T}}^{\text {miss }}$ and data-to-prediction ratio in the mono- $\gamma$ signal region. Right: 95\% CL exclusion contours in the $m_{\chi}-m_{\text {med }}$-plane. The region on the left is excluded by the perturbative limit. The relic density curve is also shown [8].
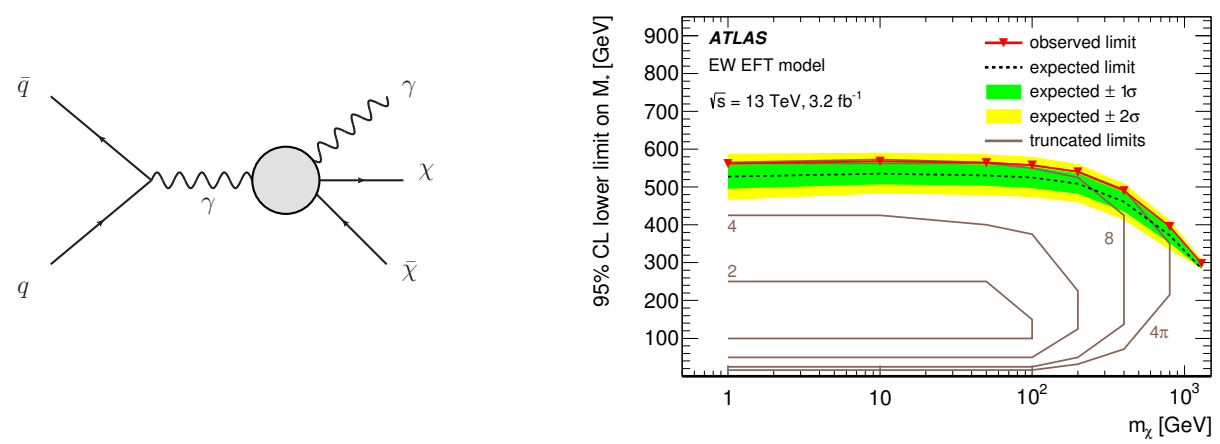

Figure 4: Left: Production of pairs of dark matter particles via an effective $\gamma \gamma \chi \bar{\chi}$ vertex. Right: 95\% CL limits on the suppression scale, $M_{*}$, for the model shown on the left. Results where EFT truncation is applied are also shown, assuming representative coupling values [8].

the process depicted in the left panel of Fig. 4 where the photon couples directly to the WIMPs via an effective vertex. The corresponding 95\% CL bounds on the suppression scale $M_{*}$ as a function of the WIMP mass are shown in the right panel of Fig. 4. The observed limit is displayed as a red line, the expected limits as a dotted black line together with the $\pm 1 \sigma$ and $\pm 2 \sigma$ uncertainty bands in green and yellow, respectively. Truncated limits for different choices of the coupling are indicated by brown lines.

\subsection{Mono-V}

A search was also performed in events with a hadronically decaying heavy vector boson [9]. The $E_{\mathrm{T}}^{\text {miss }}$ is required to be greater than $250 \mathrm{GeV}$ and a number of cuts is applied to suppress the multi-jet background. Furthermore, there has to be at least one large-radius jet (with a radius parameter of 1.0) with a $p_{\mathrm{T}}$ above $200 \mathrm{GeV}$ in the event. The jets are moreover required to be boson-tagged, i.e. their mass has to be compatible with the mass of a $W$ or $Z$ boson and they have to have a substructure indicating two sub-jets.

The dominant backgrounds are $Z+$ jets and $W+$ jets production and control regions are used to constrain them. An additional control region is defined for the $t \bar{t}$ background. Criteria used in the CR definitions are the lepton multiplicity, b-tagged jet multiplicity, mass and $E_{\mathrm{T}}^{\mathrm{miss}}$ cuts. 


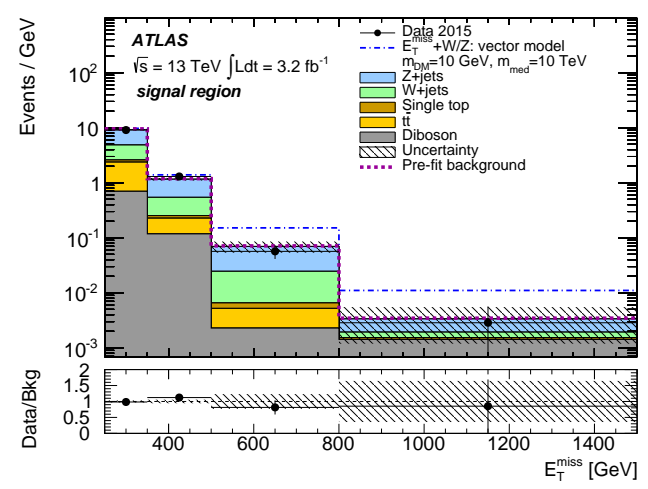

Figure 5: Distribution of $E_{\mathrm{T}}^{\text {miss }}$ in the signal region after the profile-likelihood fit to the data under the background-only hypothesis. The total background before the fit is shown as a dashed line. The lower panel shows the ratio of data to the background prediction [9].
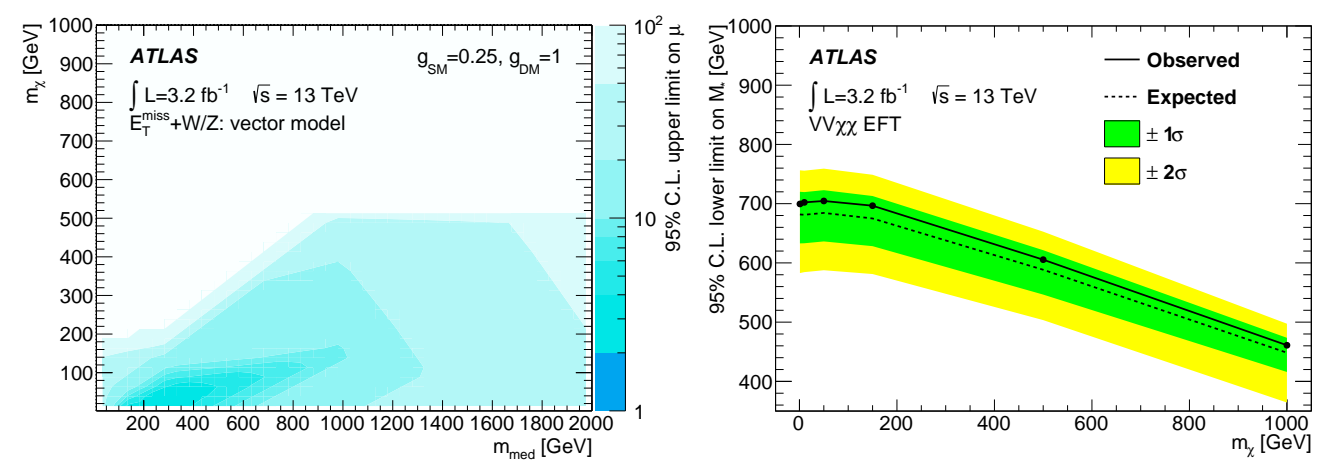

Figure 6: Left: $95 \%$ CL observed limit on the signal strength, $\mu$, for the simplified model in the $m_{\chi}-m_{\text {med }^{-}}$ plane. Right: 95\% CL limits on the suppression scale, $M_{*}$, for the $V V \chi \bar{\chi}$ model [9].

This analysis uses not only event counts but the shape of the $E_{\mathrm{T}}^{\text {miss }}$ spectrum. The $E_{\mathrm{T}}^{\text {miss }}$ distribution in the signal region is shown in Fig. 5. The dominant uncertainties are related to the modelling of the large-radius jets and amount to about $10 \%$. No significant deviation from the prediction is observed and the results are interpreted in a simplified model with a vector mediator and a $W$ or $Z$ boson from ISR as well as a model with an effective $V V \chi \chi$ vertex analogously to the mono-photon search. The left panel of Fig. 6 shows the 95\% CL limits on the signal strength for the simplified model in the $m_{\chi}-m_{m e d}$-plane. In the right panel of Fig. 6 the 95\% CL on the suppression scale of the effective model are shown as a function of the WIMP mass. They vary between $700 \mathrm{GeV}$ and $450 \mathrm{GeV}$ for the mass range considered.

\subsection{Mono-Higgs}

The discovery of a Higgs-boson in LHC run-1 has opened a new window for the search for BSM physics. With the first $13 \mathrm{TeV}$ data, ATLAS has performed several mono-Higgs searches. In the models considered, the Higgs-boson is not emitted as ISR, however, as the couplings are exceedingly small. A widely used simplified model is one with a $Z^{\prime}$-type vector mediator that radiates the Higgs, cf. top left of Fig. 7. Other simplified models in use include s-channel scalar 

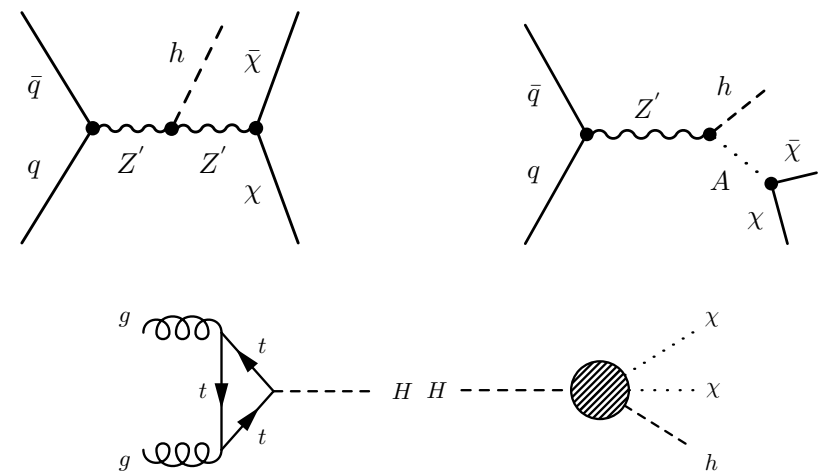

Figure 7: Feynman diagrams of models employed in the mono-H searches. Top: $Z^{\prime}$-like vector mediator on the left and $Z^{\prime}$-2HD model on the right [10], bottom: Heavy scalar 2HD model [11].

mediators, Z'-2HD (2 Higgs doublet) (cf. top right of Fig. 7) and scalar 2HD models (cf. bottom of Fig. 7). These models typically come with some additional parameters such as the $Z^{\prime} Z^{\prime} h$ coupling and mixing angles. Mono-Higgs searches are performed in several channels of Higgs decay, as outlined in the following sections.

\subsection{1 $H \rightarrow b \bar{b}$}

The mono-Higgs search with the Higgs decaying to two $b$-quarks [10] applies a cut on $E_{\mathrm{T}}^{\text {miss }}$ to be greater than $150 \mathrm{GeV}$. The missing transverse momentum $p_{\mathrm{T}}^{\text {miss }}$, which is reconstructed from the tracks in the event, has to be greater than $30 \mathrm{GeV}$. Several cuts for example on angular quantities are applied to suppress the multi-jet background. In the two signal regions, events with leptons are vetoed and two $b$-tagged jets are required. For $E_{\mathrm{T}}^{\text {miss }}>500 \mathrm{GeV}$ the Higgs-boson is boosted and the jets from the decay quarks merge - in this merged $\mathrm{SR}$ one jet with radius parameter 1.0 is required and in addition two $b$-tagged jets reconstructed from tracks. In the resolved signal region below $500 \mathrm{GeV}$ of $E_{\mathrm{T}}^{\text {miss }}$ two $b$-tagged jets with radius parameter of 0.4 are required. The $E_{\mathrm{T}}^{\text {miss }}$ spectrum with the two regions indicated is shown in the left panel of Fig. 8.

Orthogonal control regions are defined based on the number of $b$-tagged jets and leptons. A fit to the invariant mass of the di-jet pair or the large- $\mathrm{R}$ jet, respectively, is performed. The dominant uncertainties arise from $b$-tagging and theory uncertainties in the background normalisation. The total uncertainty after the fit amounts to a few \%. In the right panel of Fig. 8 the large-jet mass in the merged signal region is shown. Within the uncertainties data and simulation agree. Therefore, limits are derived on two simplified models. The left panel in Fig. 9 shows bounds on a Z'-2HD model in the plane of the $Z^{\prime}$ and $A$-boson mass. The observed (expected) limit are displayed as black solid (dashed) lines, with the green and yellow $\pm 1 \sigma$ and $\pm 2 \sigma$ uncertainty bands around the expected limit. The bounds are extended considerably beyond the run-1 result which is indicated by a black dotted line. The right panel of Fig. 9 shows the bounds on the vector mediator model in the plane of the mass of this mediator and the WIMP mass. This interpretation is new in run- 2 and the exclusion reaches up to about $1 \mathrm{TeV}$ in mediator mass.

\subsection{2 $H \rightarrow \gamma \gamma$}

For the mono-Higgs search with the Higgs decaying into two photons [11] events are selected 

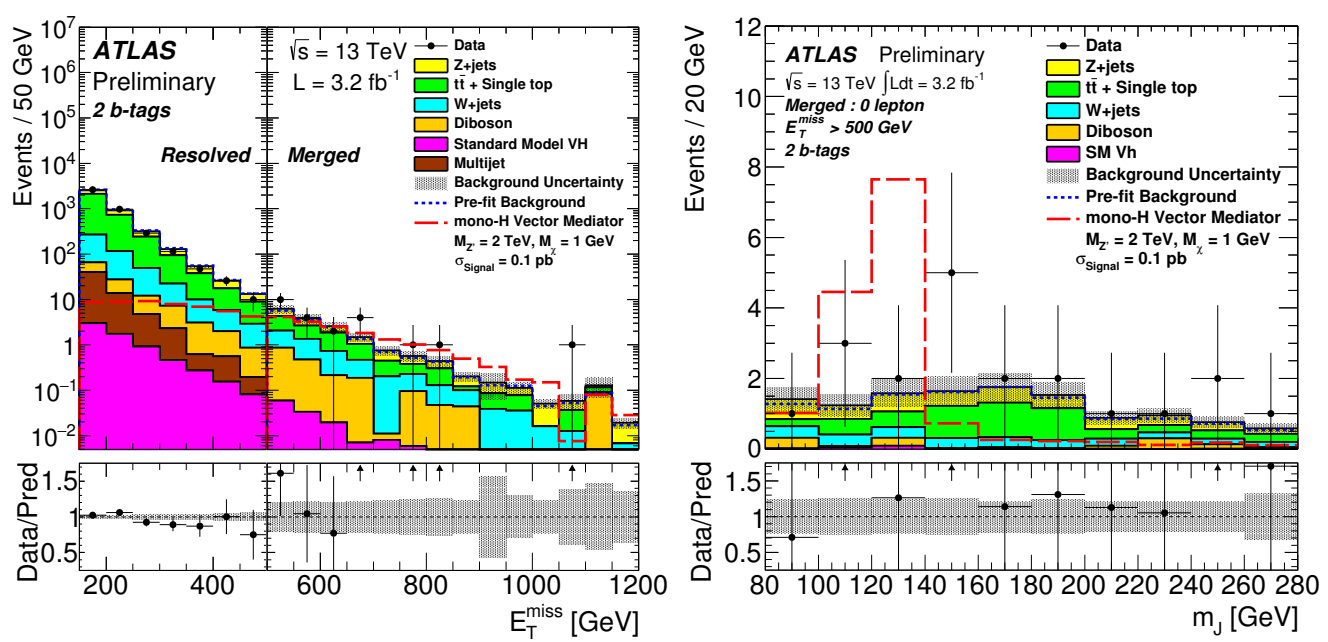

Figure 8: Left: Distribution of $E_{\mathrm{T}}^{\text {miss }}$ in the signal regions. Right: Distribution of large-jet mass, $m_{\mathrm{J}}$ in the merged signal region. The lower panels show the data-to-prediction ratio [10].
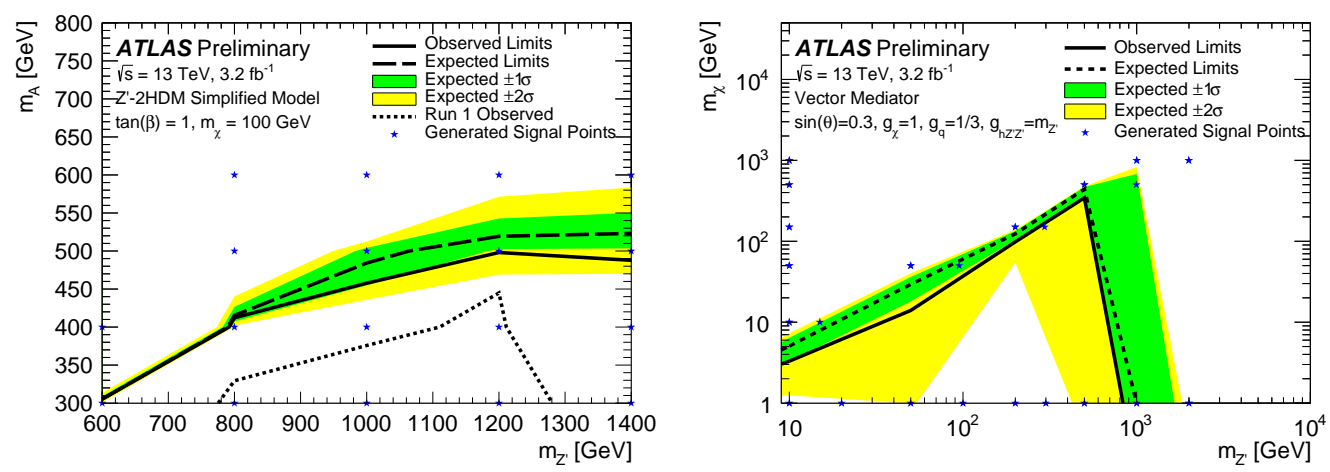

Figure 9: Exclusion contours for the $Z^{\prime}-2 \mathrm{HDM}$ in the $m_{\mathrm{A}}-m_{Z^{\prime}}$-plane (left) and the vector mediator model in the $m_{\chi}-m_{Z}$-plane (right) [10].

using a di-photon trigger. Two photons are required with an invariant mass between $105 \mathrm{GeV}$ and $160 \mathrm{GeV}$. Events are further categorised into four regions based on cuts on the $E_{\mathrm{T}}^{\text {miss }}$, the diphoton transverse momentum $p_{\mathrm{T}}^{\gamma \gamma}$ and the sum of the transverse momenta of the photons and jets in the events. A simultaneous fit is performed to all four regions. The highest sensitivity to both models considered is obtained from a region with $E_{\mathrm{T}}^{\text {miss }}>100 \mathrm{GeV}$ and $p_{\mathrm{T}}^{\gamma \gamma}>100 \mathrm{GeV}$. The diphoton invariant mass in this region is shown in Fig. 10. The largest uncertainties are in the selection of the $\gamma \gamma$ vertex and $E_{\mathrm{T}}^{\text {miss }}$. Limits on the cross section times branching ratio for the vector mediator and the heavy scalar model are derived and presented in Fig. 11.

\subsection{3 $H \rightarrow Z Z$}

In the context of the SM measurement of the Higgs-boson decaying into four leptons (via two Z-bosons) [12] a mono-Higgs search for DM was performed as well. Events with a leptonquadruplet invariant mass between $110 \mathrm{GeV}$ and $140 \mathrm{GeV}$ are considered. Fig. 12 (left) shows the $E_{\mathrm{T}}^{\text {miss }}$ distribution in these events. The region of $E_{\mathrm{T}}^{\text {miss }}<100 \mathrm{GeV}$ is used as a control region, the 


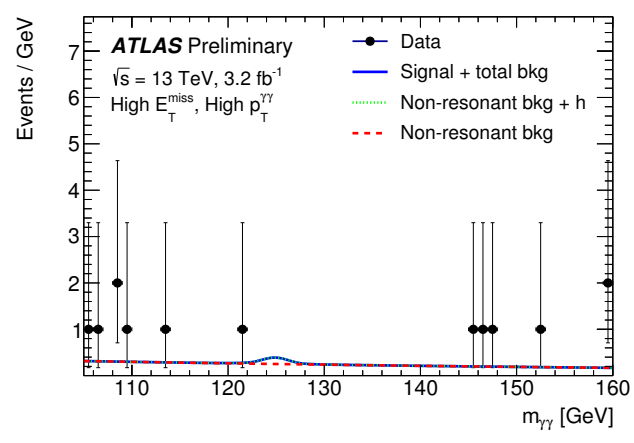

Figure 10: Distribution of the diphoton invariant mass in the most sensitive event category. [11]
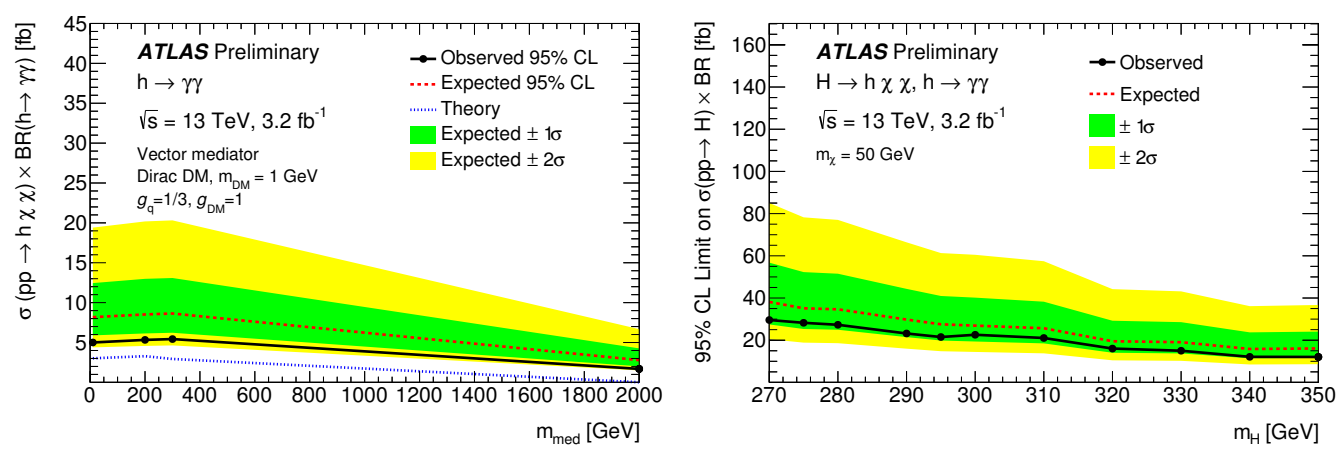

Figure 11: $95 \%$ CL limits on the $\sigma \times B R$ for the vector mediator model (left) and the heavy scalar 2HDM (right) [11].

signal region is defined by $E_{\mathrm{T}}^{\text {miss }}>100 \mathrm{GeV}$. Large uncertainties arise due to the jet energy scale (up to $60 \%$ ). No significant excess is observed and limits on the cross section times branching ratio on the vector mediator model are derived and shown in the right panel of Fig. 12.
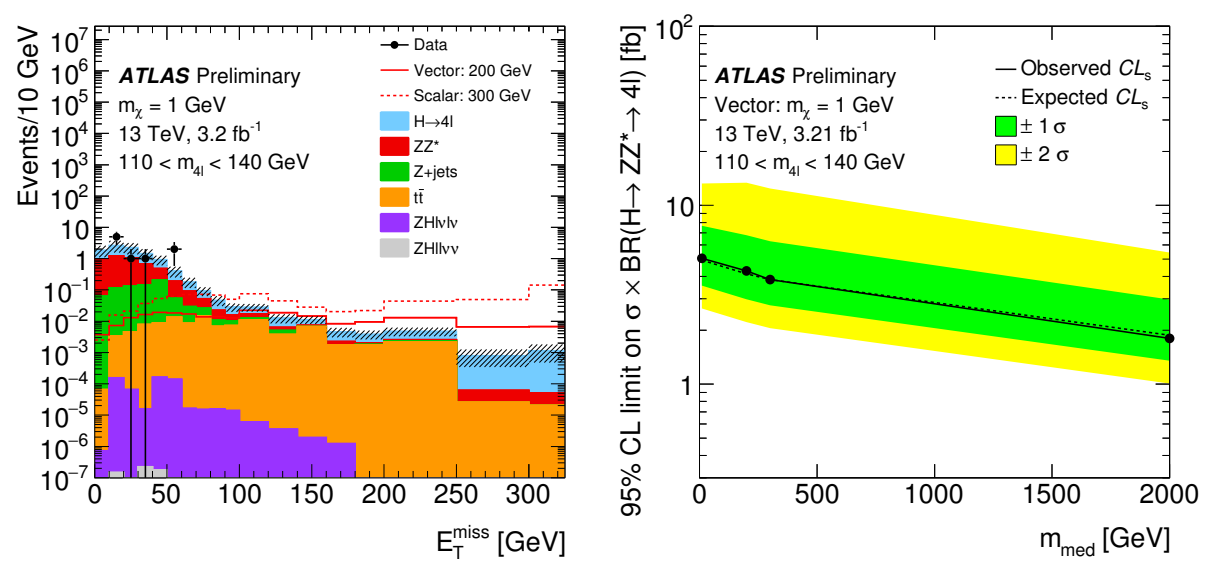

Figure 12: Left: Distribution of $E_{\mathrm{T}}^{\text {miss }}$ for events in the Higgs-mass window. Right: 95\% CL limits on the $\sigma \times B R$ for a vector mediator model [12]. 


\section{Summary}

ATLAS has performed a suite of mono-X searches with the $3.2 \mathrm{fb}^{-1}$ of $13 \mathrm{TeV}$ data collected in 2015. None of the searches observed a significant excess. The interpretations are done using simplified models instead of the effective theory that was used in run-1 wherever possible.

\section{References}

[1] Planck Collaboration, Planck 2015 results. I. Overview of products and scientific results, 2015, arXiv:1502.01582

[2] The ATLAS Collaboration, The ATLAS Experiment at the CERN Large Hadron Collider, JINST 3 (2008) S08003, doi: 10.1088/1748-0221/3/08/S08003

[3] Goodman, Jessica et al., Constraints on Light Majorana dark Matter from Colliders, Phys. Lett. $\mathbf{6 6 9 5}$ (2011) pp. 185-188, doi: 10.1016/j.physletb.2010.11.009

[4] Goodman, Jessica et al., Constraints on Dark Matter from Colliders, Phys. Rev, D82 (2010), p. 116010, doi: 10.1103/PhysRevD.82.116010

[5] Abercrombie, Daniel et al., Dark Matter Benchmark Models for Early LHC Run-2 Searches: Report of the ATLAS/CMS Dark Matter Forum, 2015, arxiv:1507.00966

[6] Boveia, Antonio et al., Recommendations on presenting LHC searches for missing transverse energy signals using simplified s-channel models of dark matter, 2016, arxiv:1603.04156

[7] The ATLAS Collaboration, Search for new phenomena in final states with an energetic jet and large missing transverse momentum in pp collisions at $\sqrt{s}=13$ TeV using the ATLAS detector, accepted by PRD, preprint: arxiv:1604.07773

[8] The ATLAS Collaboration, Search for new phenomena in events with a photon and missing transverse momentum in pp collisions at $\sqrt{s}=13 \mathrm{TeV}$ with the ATLAS detector, JHEP 06 (2016) 059, doi: 10.1007/JHEP06(2016)059

[9] The ATLAS Collaboration, Search for dark matter produced in association with a hadronically decaying vector boson in pp collisions at $\sqrt{(s)}=13$ TeV with the ATLAS detector, submitted to PLB, preprint: arxiv: 1608.02372

[10] The ATLAS Collaboration, Search for Dark Matter in association with a Higgs boson decaying to b-quarks in pp collisions at $\sqrt{s}=13 \mathrm{TeV}$ with the ATLAS detector, ATLAS-CONF-2016-019, https://cds.cern.ch/record/2142777

[11] The ATLAS Collaboration, Search for new phenomena in events with missing transverse momentum and a Higgs boson decaying to two photons in p p collisions at $\sqrt{s}=13 \mathrm{TeV}$ with the ATLAS detector, ATLAS-CONF-2016-011, https://cds.cern.ch/record/2139812

[12] The ATLAS Collaboration, Measurements of the Higgs boson production cross section at 7, 8 and 13 $\mathrm{TeV}$ centre-of-mass energies and search for new physics at $13 \mathrm{TeV}$ in the $H \rightarrow Z Z^{*} \rightarrow \ell^{+} \ell^{-} \ell^{\prime+} \ell^{\prime-}$ final state with the ATLAS detector, ATLAS-CONF-2015-059, https://cds.cern.ch/record/21148252 